

LEM | Laboratory of Economics and Management

Institute of Economics

Scuola Superiore Sant'Anna

Piazza Martiri della Libertà, 33 - 56127 Pisa, Italy ph. +3905088.33 .43$

institute.economics@sssup.it

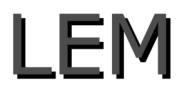

Working Paper Series

\title{
Decidability in complex social choices
}

\author{
Luigi Marengo" \\ Davide Pirino" \\ Simona Settepanellaף \\ Akimichi Takemura ${ }^{\circ}$ \\ 'Institute of Economics and LEM, Scuola Superiore Sant'Anna, Pisa, Italy \\ ${ }^{\circ}$ Department of Mathematical Informatics, University of Tokyo, Japan
}




\title{
Decidability in complex social choices
}

\author{
Luigi Marengo* Davide Pirino ${ }^{\dagger}$ Simona Settepanella \\ Akimichi TAKEMURA ${ }^{\S}$
}

July 5, 2012

\begin{abstract}
Recently, Marengo and Settepanella (2010) introduced a model of social choice among bundles of interdependent elements. In this paper we prove that their voting model is highly decidable, i.e. a group of agents that agrees to use such voting process has an high probability to reach a final decision. We also better qualify the degree of manipulability of such a final decision, showing that it is independent not only from the agenda, but also from the initial condition. Therefore we show that the Marengo and Settepanella (2010) model has nice properties of decidability and can be fruitfully used both for normative and positive analyzes of collective choices among complex interdependent elements.
\end{abstract}

\section{Keywords:}

Social rule, object, optimum, probability, tournament

MSC (2010): 05C20, 05C38, 91B10, 91B12, 91B14.

JEL Classification: D03, D71, D72.

${ }^{*}$ L.E.M, Scuola Superiore Sant'Anna, Pisa, Italy, l.marengo@sssup.it

${ }^{\dagger}$ L.E.M, Scuola Superiore Sant'Anna, Pisa, Italy, d.pirino@sssup.it.

${ }^{\ddagger}$ L.E.M, Scuola Superiore Sant’Anna, Pisa, Italy, s.settepanella@sssup.it. Dr. Settepanella was partially supported by the Institute for New Economic Thinking, INET inaugural grant $\sharp 220$

${ }^{\S}$ Department of Mathematical Informatics, Graduate School of Information Science and Technology, The University of Tokyo 


\section{Introduction}

In his seminal 1951 book Kenneth Arrow laid down the foundations of modern social choice theory (Arrow, 1951), a discipline whose applications span from social ethics, to political sciences and to economics (see, among others, Elster and Hylland, 1986; Taylor, 2005; Feldman and Serrano, 2006; Gaertner, 2006, for a review) and whose central aim is to study the aggregation of preferences.

Social choice theory usually assumes that agents are faced with a set of exogenously given and mutually exclusive alternatives. These alternatives are "simple", in the sense that either they are one-dimensional objects or, when they are multidimensional, they are points in some portion of the homogeneous $\mathbb{R}^{n}$ space and lack any kind of internal structure.

However, in most real life situations, choices are made among "complex" objects, made of many interdependent elements, and preferences over such object are usually dependent upon their internal structure, i.e. upon the specific ways in which elements are combined. Consider for instance a typical textbook example of social choice, where a group of friends has to decide what to do in the evening. The choice set is usually presented as a set of given and mutually exclusive alternatives, e.g. \{movie, concert, restaurant, dinner at home,... $\}$. However, at a closer scrutiny, these alternatives are nothing but labels for bundles of elements (e.g. with whom, where, when, movie genre, type of food...) and the preferences are unlikely to be expressed before the labels get specified in their constituting elements. Moreover, preferences on specific elements are usually interdependent and non-separable. For instance, one's preferences on the 'with whom' element are not usually separable from those on the 'what we do' element.

Other examples can be candidates and parties in political elections (which stand for complex bundles of interdependent policies and personality traits), packages of policies on which committees and boards are called upon to decide and small group processes, such as Supreme Court or legislative decisions.

Multidimensional voting models indeed have been already widely studied (Kramer, 1972; Shepsle, 1979; Denzau and Mackay, 1981; Enelow and Hinich, 1983, e.g.), however the literature has only modeled situations in which choice takes place over multiple dimensions. A different approach is developed by Marengo and Settepanella (2010) (from now on we will refer to it as MAST). Here, the authors develop a model of social choice among bundles of elements that they call objects.

What they prove is that by bundling in different ways the same set of 
constituent elements (or features, as they call them), the social outcome (obtained for instance by sincere majority voting) changes. MAST presents a model in which social choice takes place by means of an algorithmic procedure (e.g. majority voting) whereby a population of agents aggregate their heterogeneous individual preferences. This procedure can end up in a global (or classical) optimum, i.e. an alternative that is socially preferred to all the others, or in a cycle, or in a local optimum. The latter is an alternative that is socially preferred to all the others that, according to the social choice procedure, are accessible from it. Local optima depend, in general, upon the particular bundling of elements and upon the initial status quo, i.e. the alternative which the social choice process starts from, but they can also be independent from the initial status quo and, in that case, are called u-local optima (Amendola and Settepanella, 2012).

It turns out that in social choice problems there exists a fundamental trade-off between decidability (i.e. the possibility of reaching some social optimum in a feasible time) and non manipulability (i.e. the convergence of the social decision process to a unique global outcome that does not depend upon initial conditions, object construction and agenda).

In MAST this trade-off is strongly related to the probability of having at least one local or u-local optimum. Using a properly designed software, called FOSoR ${ }^{1}$, Amendola and Settepanella (2012) and Amendola, Marengo, and Settepanella (2012) show that such probabilities can be computed numerically.

Their computations proved that, despite the fact that local optima and $\mathrm{u}$-local optima are not optima in the classical meaning, they are a good compromise in the aforementioned trade-off. Indeed the introduction of objects (as presented in MAST) has a twofold effect: first, it decomposes the search space into quasi-separable subspaces (see Simon, 1982) simplifying the computational task and, second, the probability to get a (u-) local optimum is far bigger than that of getting a classical one.

In this paper we make a step forward and we prove that the probability to get at least a local optimum when each feature has two possible outcomes (the yes/no or $0 / 1$ case) is always greater than $60 \%$, i.e. the decidability in this case is always very high, and, moreover, is independent from the number of alternatives, while, in the classical framework, the probability to get an optimum goes to zero rapidly. In addition, we prove that if the number of

\footnotetext{
${ }^{1}$ http://www.dm.unipi.it/ amendola/files/software/fosor/
} 
alternatives is "big enough", then the probability that a local optimum is a $\mathrm{u}$-local optimum is almost 1 . As a direct consequence, we prove that $\mathrm{u}$-local optima are attainable with a high probability.

These results shows that u-local optima can be seen as striking an efficient balance in the decidability vs. non manipulability trade-off.

The paper is organized as follows: in Section 1 we recall some basic notions on graphs and tournaments. In Section 2 we sketch the fundamental ideas of social choice theory, we describe a simplified version of the MAST model, we recall their main results and we end the Section with the definition of $u$-local optimum. Section 3 is dedicated to the exposition and proof of the main result while Section 4 concludes.

\section{Graphs and tournaments}

We recall here some basic notions of graph and tournament theory just to introduce our notation, for a more complete discussion we refer the reader to Chartrand and Lesniak (2005) and Moon (1968).

Graphs We will only employ directed simple graphs. Hence, throughout the paper, a graph will be a pair $(\mathcal{V}, \mathcal{E})$, where $\mathcal{V}$ is the set of nodes and $\mathcal{E}$ is the set of arcs, such that each pair of nodes $\{p, q\}$ is connected by at most one oriented arc (either $\overrightarrow{p q}$ or $\overleftarrow{p q}$ ). If the arc $\overrightarrow{p q}$ (or $\overleftarrow{q p}$ ) is in $\mathcal{E}$, the node $p$ is said to dominate $q$. A sub-graph of $(\mathcal{V}, \mathcal{E})$ is a graph $\left(\mathcal{V}^{\prime}, \mathcal{E}^{\prime}\right)$ such that $\mathcal{V}^{\prime} \subset \mathcal{V}$ and $\mathcal{E}^{\prime} \subset \mathcal{E}$.

A domination path $D P(p, q)$ from $p$ to $q$ is a sequence of arcs of the type $\overleftarrow{p p_{1}}, \overleftarrow{p_{1} p_{2}}, \ldots, \overleftarrow{p_{k} q}$. A cycle is a domination path $D P(p, p)$ from $p$ to itself. The length of a domination path is the number of arcs it contains; a cycle of length $k$ is called $k$-cycle.

Tournaments A tournament is a complete directed graph (i.e. each pair of nodes $\{p, q\}$ is connected by an arc). By $\mathcal{T}$ we will always denote a tournament with $M$ nodes. A sub-tournament of $\mathcal{T}$ is a sub-graph of $\mathcal{T}$ that is itself a tournament.

A tournament is said to be reducible if it is possible to partition its nodes into two non-empty subsets $\mathcal{V}_{1}$ and $\mathcal{V}_{2}$ in such a way that all the nodes in $\mathcal{V}_{1}$ dominate all the nodes in $\mathcal{V}_{2}$; otherwise it is called irreducible. A tournament is irreducible if and only if each pair of nodes is contained in a cycle. 
Remark 1.1. Let us remark that there is no bound on the length of the cycle that contains the two nodes, nevertheless every node of an irreducible tournament is contained in a $k$-cycle for all $k=3,4, \ldots, M$. Hence there is a cycle that contains all nodes of a irreducible tournament.

An irreducible component $\mathcal{T}_{i}$ of $\mathcal{T}$ is a maximal irreducible sub-tournament of $\mathcal{T}$. The nodes of these irreducible components form a partition of the nodes of $\mathcal{T}$. Moreover, all the nodes of a component $\mathcal{T}_{i}$ either dominate or are dominated by all the nodes of another component $\mathcal{T}_{j}$.

The probability $\operatorname{Prob}(M)$ that a tournament with $M$ nodes is irreducible can be computed recursively by the formula:

$$
\operatorname{Prob}(M)=1-\sum_{i=1}^{M-1}\left(\begin{array}{c}
M \\
i
\end{array}\right) \frac{\operatorname{Prob}(i)}{2^{i(M-i)}}, \text { with } \operatorname{Prob}(1)=1 .
$$

The values of $\operatorname{Prob}(M)$ for $M \leqslant 16$ are given in Figure 1 . As $M$ tends to infinity, $\operatorname{Prob}(M) \rightarrow 1$ and $\operatorname{Prob}(M) \sim 1-\frac{M}{2^{M-2}}$ holds.

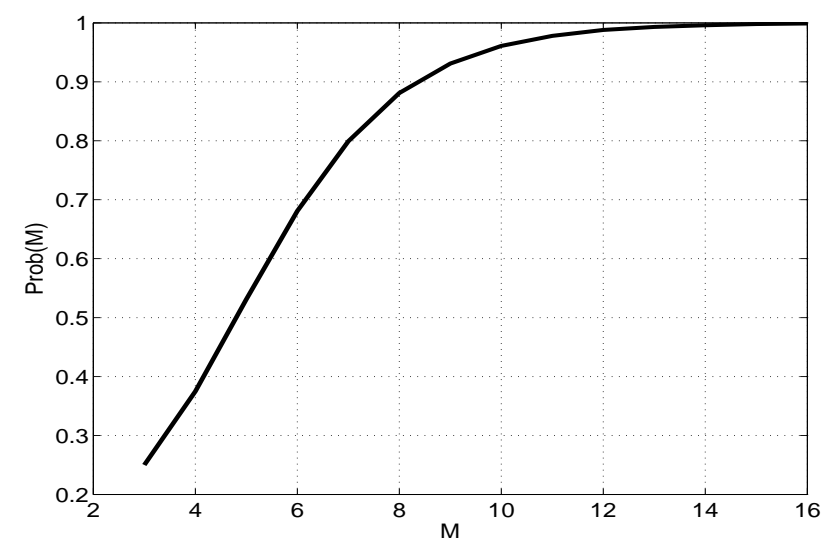

Figure 1: The probability that a tournament is irreducible as a function of $M$.

\section{Definitions and structure of the model}

Social decision rules Consider a population of $\nu$ agents. Each agent $i$ is characterized by a system of transitive preferences $\succeq_{i}$ over the set of social 
outcomes $X$. The set of systems of transitive preferences $\succeq$ is denoted by $\mathcal{P}$. A social decision rule $\mathcal{R}$ is a function:

$$
\begin{array}{ccc}
\mathcal{R}: & \mathcal{P}^{\nu} & \longrightarrow \overline{\mathcal{P}} \\
\left(\succeq_{1}, \ldots, \succeq_{\nu}\right) & \longmapsto \succeq_{\mathcal{R}\left(\succeq_{1}, \ldots, \succeq_{\nu}\right)}
\end{array}
$$

which determines a system of social preferences or social rule $\succeq_{\mathcal{R}\left(\succeq_{1}, \ldots, \succeq_{\nu}\right)}$ from the preferences of $\nu$ individual agents. With $\overline{\mathcal{P}}$ we denote the set of systems of (non-necessarily transitive) social preferences; as a matter of fact, we note that the social rule $\succeq_{\mathcal{R}\left(\succeq_{1}, \ldots, \succeq_{\nu}\right)}$ is not, in general, transitive anymore.

If $\Delta$ is the diagonal of the Cartesian product $X \times X$, the element $\succeq_{\mathcal{R}} \in \overline{\mathcal{P}}$ defines a subset

$$
\mathcal{Y}_{1, \succeq_{\mathcal{R}}}=\left\{(x, y) \in X \times X \backslash \Delta \mid x \succeq_{\mathcal{R}} y\right\}
$$

and the set of relevant social outcomes

$$
\mathcal{Y}_{0, \succeq_{\mathcal{R}}}=\left\{x \in X \mid \exists y \in X \text { such that }(x, y) \in \mathcal{Y}_{1, \succeq \mathcal{R}} \text { or }(y, x) \in \mathcal{Y}_{1, \succeq_{\mathcal{R}}}\right\} .
$$

If $\mathcal{Y}_{0, \succeq_{\mathcal{R}}}$ is the whole $X$, the social rule is said to be complete. A complete social rule is said to be strict if for each pair of social outcomes $x$ and $y$ the two conditions $x \succeq_{\mathcal{R}} y$ and $y \succeq_{\mathcal{R}} x$ are mutually exclusive (i.e. either the social outcome $x$ is preferred to the social outcome $y$ or the converse holds). For the sake of simplicity, we will consider only strict social rules. This restriction is almost always unnecessary, but it simplifies the presentation. Therefore, from now on, we will consider a complete and strict system of preferences, denoted by $\succ$.

The graph The sets $\mathcal{Y}_{0, \succ}$ and $\mathcal{Y}_{1, \succ}$ correspond, respectively, to the sets of nodes and arcs of a graph $\mathcal{Y}_{\succ}$. Two nodes $x$ and $y$ in $\mathcal{Y}_{0, \succ}$ are connected by an arc $\overrightarrow{x y}$ if $(x, y) \in \mathcal{Y}_{1, \succ}$ or $\overrightarrow{y x}$ if $(y, x) \in \mathcal{Y}_{1, \succ}$. Note that the completeness assumption on social rules guarantees that the graph $\mathcal{Y}_{\succ}$ is connected.

A cycle

$$
\overrightarrow{x_{1} x_{2}}, \overrightarrow{x_{2} x_{3}}, \ldots, \overrightarrow{x_{h} x_{1}},
$$

in the graph $\mathcal{Y}_{\succ}$ corresponds to a cycle $\grave{a}$ la Condorcet-Arrow, i.e. to the sequence

$$
x_{1} \succ x_{2} \succ \cdots \succ x_{h} \succ x_{1}
$$


Features Let $F=\left\{f_{1}, \ldots, f_{n}\right\}$ be a bundle of elements, called features, the $i$-th of which takes $m_{i}$ values, i.e. $f_{i} \in\left\{0,1,2, \ldots, m_{i}-1\right\}$ with $i=1, \ldots, n$. Denote by $m=\left(m_{1}, \ldots, m_{n}\right)$ the multi-index of the numbers of values of the features. Hence, a social outcome (or alternative) can be denoted by $v_{1}, \cdots v_{n}$, with $0 \leqslant v_{i}<m_{i}$. From now on the set of all social outcomes will be denoted by $X$. The cardinality of $X$ is $\prod_{i=1}^{n} m_{i}$ and will be denoted by $M$.

Objects schemes Given a non-empty subset $I \subseteq\{1, \ldots, n\}$, the object $\mathcal{A}_{I}$ is the set

$$
\mathcal{A}_{I}=\left\{f_{i} \mid i \in I\right\} .
$$

The complement of a set $I$ in $\{1, \ldots, n\}$ will be denoted as $I^{c}$. An objects scheme is a set of objects $A=\left\{\mathcal{A}_{I_{1}}, \ldots, \mathcal{A}_{I_{k}}\right\}$ such that $\bigcup_{j=1}^{k} I_{j}=\{1, \ldots, n\}$. Note that the sets $I_{j}$ may have non-empty intersection.

Neighbors of a social outcome Let $A=\left\{\mathcal{A}_{I_{1}}, \ldots, \mathcal{A}_{I_{k}}\right\}$ be an objects scheme. A social outcome $y$ is said to be a preferred neighbor of a social outcome $x$ with respect to an object $\mathcal{A}_{I_{h}} \in A$ if the following conditions hold:

1) $y \succ x$,

2) $y\left(\mathcal{A}_{I_{h}^{c}}\right)=x\left(\mathcal{A}_{I_{h}^{c}}\right)$, i.e. in $x$ and $y$ the features $f_{j} \notin A_{I_{h}}$ have the same value,

3) $y\left(\mathcal{A}_{I_{h}}\right) \neq x\left(\mathcal{A}_{I_{h}}\right)$, i.e. $x$ and $y$ have different values for at least one feature $f_{j} \in A_{I_{h}}$.

The set of all preferred neighbors of the social outcome $x$ with respect to $\mathcal{A}_{I_{h}} \in A$ is denoted by $\Phi\left(x, \mathcal{A}_{I_{h}}\right)$. The set of all preferred neighbors of the social outcome $x$ with respect to an object scheme $A$ is denoted by $\Phi(x, A)=\bigcup_{j=1}^{k} \Phi\left(x, \mathcal{A}_{I_{j}}\right)$.

A social outcome $y \in \Phi\left(x, \mathcal{A}_{I_{h}}\right)$ is said to be a best neighbor of a social outcome $x$ with respect to an object $\mathcal{A}_{I_{h}} \in A$ if

$$
y \succ w \quad \forall w \in \Phi\left(x, \mathcal{A}_{I_{h}}\right) .
$$

The set of all best neighbors of the social outcome $x$ with respect to $\mathcal{A}_{I_{h}} \in A$ is denoted by $B\left(x, \mathcal{A}_{I_{h}}\right)$. When preferences are strict, either $B\left(x, \mathcal{A}_{I_{h}}\right)$ is empty or $B\left(x, \mathcal{A}_{I_{h}}\right)$ contains one social outcome only. 
The set of all best neighbors of the social outcome $x$ is denoted by $B(x, A)=\bigcup_{j=1}^{k} B\left(x, \mathcal{A}_{I_{j}}\right)$.

A domination path $D P(x, y, A)$ through $A$, starting from $x$ and ending in $y$, is a sequence of best neighbors with respect to objects in $A$, i.e. a sequence

$$
x=x_{0} \prec x_{1} \prec \cdots \prec x_{s}=y
$$

such that there exist objects, not necessarily distinct, $\mathcal{A}_{I_{h_{1}}}, \ldots, \mathcal{A}_{I_{h_{s}}} \in A$ with $x_{i} \in B\left(x_{i-1}, \mathcal{A}_{I_{h_{i}}}\right)$ for all $1 \leqslant i \leqslant s$.

A social outcome $y$ is said to be reachable from $x$ with respect to an objects scheme $A$ if there exists a domination path $D P(x, y, A)$.

A social outcome $x$ is said to be a local optimum for $A$ if $\Phi(x, A)$ is empty.

Voting System A domination path is said to be maximal if it ends in either a local optimum or a limit domination cycle: more precisely, either $x_{s}$ is a local optimum or $x_{s-t}$ belongs to $B\left(x_{s}, \mathcal{A}_{I_{h_{s}+1}}\right)$, where $h_{s}$ is the remainder of the division of $s-1$ by $t$.

Let $A=\left\{\mathcal{A}_{I_{1}}, \ldots, \mathcal{A}_{I_{k}}\right\}$ be an objects scheme. An agenda $\alpha$ of $A$ is an ordered $t$-uple of indices $\left(h_{1}, \ldots, h_{t}\right)$ with $t \geq k$ such that $\left\{h_{1}, \ldots, h_{t}\right\}=$ $\{1, \ldots, k\}$. An agenda $\alpha$ states the order in which the objects $\mathcal{A}_{I_{i}}$ are decided upon (see MAST).

The voting process consists in moving from an initial social outcome $x_{0}$, called status quo, along the maximal path through a fixed object scheme $A$, ordered by an agenda $\alpha$. If the maximal path ends up in a local optimum, then this will be the preferred choice of the society.

Remark 2.1. In MAST it is proved that the local optimum is independent from the choice of the agenda $\alpha$.

Basin of attraction The basin of attraction $\Psi(x, A)$ of a social outcome $x$ with respect to an objects scheme $A$ is the set of the social outcomes $y$ such that there exists a maximal domination path $D P(y, x, A)$ that ends up in $x$.

Remark 2.2. Note that $\Psi(x, A)$ is empty if and only if $x$ is not a local optimum for $A$.

Characterization of local optima We will say that $x$ and $y$ are separated by the feature $f$ if the value of the feature $f$ of $y$ differs from that of the 
feature $f$ of $x$. The prominent distance $d_{p}(x, y)$ is the number of features that separate $x$ and $y$. A social outcome $z$ is said to be free if and only if it is a local optimum for at least an objects scheme $A$.

In MAST it is proved that

$$
z \text { is free } \Leftrightarrow d_{p}(w, z)>1 \forall w \succ z \text {. }
$$

Namely, it exists an objects scheme $A$ such that a social outcome $z$ is a local optimum if and only if any social outcome $x$ such that $d_{p}(x, z)=1$ belongs to the basin of attraction $\Psi(z, A)$.

Let us remark that if all features take only the two values 0 and 1 , then the prominent distance is exactly the well known Hamming distance.

Universal basin of attraction and $\mathbf{u}$-local optima Let $\Pi\left(\mathcal{A}_{n, m}\right)$ be the set of all possible objects schemes in $\mathcal{A}_{n, m}$. The universal basin of attraction of a social outcome $z \in X$ is the set

$$
\Psi(z)=\bigcup_{A \in \Pi\left(\mathcal{A}_{n, m}\right)} \Psi(z, A),
$$

i.e. the set of all the social outcomes $x$ such that there exists an objects scheme through which there is a domination path starting from $x$ and ending up in $z$. The universal basin of attraction of the social outcome $z$ is non-empty if and only if $z$ is free.

Definition 2.3. A social outcome $z$ is said to be an u-local optimum if its universal basin of attraction $\Psi(z)$ is the whole set of social outcomes $X$.

Remark 2.4. A u-local optimum is, by definition, independent from the status quo of the voting process.

\section{Probability of Local Optima}

In this section we will compute the probability to have a local optimum or a u-local optimum showing that such a probability is large enough, i.e. we show that, with a reasonable confidence, the social choice process can indeed converge to some "acceptable", though manipulable, choice. What is shown below is that, in the MAST framework when $m_{i}=2$ for all features, that is 
$f_{i} \in\{0,1\}$, a locally optimal outcome is actually reached in more than $60 \%$ of the cases, even for a large number of alternatives.

From now on we indicate with $\operatorname{Prob}_{n}(z)$ the probability that a randomly chosen $z$ (i.e. sampled from the uniform distribution over the set of social outcomes) is a local optimum, when the number of features is $n$. Similarly, we indicate with $\operatorname{Prob}_{n}(z \wedge w)$ the probability that two randomly chosen social outcomes $z$ and $w, z \neq w$, are simultaneously local optima.

The probability $\operatorname{Prob}_{n}(z)$ is given by the quotient between the number of the graphs with $M$ nodes and with $\sum_{i=1}^{n} m_{i}-n$ fixed arcs, and the number of all the graphs with $M$ nodes, i.e.

$$
\operatorname{Prob}_{n}(z)=\frac{2^{\left(\begin{array}{c}
M \\
2
\end{array}\right)-\left(\sum_{i=1}^{n} m_{i}-n\right)}}{2^{\left(\begin{array}{c}
M \\
2
\end{array}\right)}}=\frac{1}{2^{\sum_{i=1}^{n} m_{i}-n}},
$$

that is $\operatorname{Prob}_{n}(z)=\frac{1}{2^{n}}$ when $m_{i}=2$ for all $i$.

Remark 3.1. In the classical social choice framework a given social outcome $z$ is an optimum if and only if it dominates all the other social outcomes. Therefore, the probability $P(z)$ that a randomly chosen social outcome $z$ is an optimum for a social rule on $M$ social outcomes is given by the quotient between the number of graphs with $M-1$ nodes and the number of graphs with $M$ nodes, i.e.

$$
P(z)=\frac{2^{\left(\begin{array}{c}
M-1 \\
2
\end{array}\right)}}{2^{\left(\begin{array}{c}
M \\
2
\end{array}\right)}}=\frac{1}{2^{M-1}} .
$$

It is clear that, if $n$ is greater than $1, \operatorname{Prob}_{n}(z) \gg P(z)$, i.e. the probability for a randomly chosen social outcome to be a local optimum is far greater than the probability to be the optimum in the classical framework.

In order to prove the main result of this paper we need the following Lemma.

Lemma 3.2. Let $z$ and $w, z \neq w$, be two randomly chosen social outcomes. The following holds:

$$
\lim _{n \rightarrow \infty} \frac{\operatorname{Prob}_{n}(z \wedge w)}{\operatorname{Prob}_{n}(z) \cdot \operatorname{Prob}_{n}(w)}=1 .
$$

Proof. Write $\operatorname{Prob}_{n}(z \wedge w)$ as

$$
\operatorname{Prob}_{n}(z \wedge w)=\operatorname{Prob}_{n}(z \mid w) \cdot \operatorname{Prob}_{n}(w),
$$


where the unconditional probability $\operatorname{Prob}_{n}(w)$ is given by equation (2). The probability that $z$ is a local optimum, given that $w$ is a local optimum, is easily decomposed as:

$$
\operatorname{Prob}_{n}(z \mid w)=0 \cdot \operatorname{Prob}_{n}\left(d_{p}(z, w)=1\right)+\frac{1}{2^{n}} \cdot \operatorname{Prob}_{n}\left(d_{p}(z, w)>1\right),
$$

where the zero on the first term comes from the fact that if the prominent distance between two social outcomes is 1 and one of the two is a local optimum then the other cannot be a local optimum itself (see condition in equation 1). Now, note that the number of social optima with prominent distance equal to 1 is obtained by summing $n$ (the total number of prominent neighbors for each social outcome) for $2^{n}$ times (the total number of social outcomes) and dividing the final result by two (every arc is counted twice). Considering that the total number of pairs of social outcomes (or the total number of edges of the complete graph) is $\frac{2^{n}\left(2^{n}-1\right)}{2}$ we get:

$$
\begin{aligned}
\operatorname{Prob}_{n}\left(d_{p}(z, w)>1\right) & =1-\operatorname{Prob}_{n}\left(d_{p}(z, w)=1\right)= \\
& =1-\frac{\frac{n 2^{n}}{2}}{\frac{2^{n}\left(2^{n}-1\right)}{2}}= \\
& =1-\frac{n}{2^{n}-1} \rightarrow 1 .
\end{aligned}
$$

Hence:

$$
\operatorname{Prob}_{n}(z \mid w) \sim \operatorname{Prob}_{n}(z)
$$

or:

$$
\operatorname{Prob}_{n}(z \wedge w) \sim \operatorname{Prob}_{n}(z) \cdot \operatorname{Prob}_{n}(w),
$$

which is our thesis.

The above Lemma essentially prove that the events " $z$ is a local optimum" and " $w$ is a local optimum" are asymptotically independent.

We can now state and prove the theorem that formalizes our main result: 
Theorem 3.3. Let $X$ be the set of possible social outcomes given by a bundle of features $F=\left\{f_{1}, \ldots, f_{n}\right\}$ such that $f_{i}$ belongs to $\{0,1\}$ for $i=1, \ldots, n$. Let $M$ be the number of social outcomes and let $K(M)$ denote the number of local-optima. Then for each $k=0,1, \ldots$ we have

$$
\lim _{n \rightarrow \infty} \operatorname{Prob}(K(M)=k)=e^{-1} \frac{1}{k !} .
$$

In particular the probability to have at least one local optimum converges to:

$$
\lim _{n \rightarrow \infty} \operatorname{Prob}(K(M) \geq 1)=1-\lim _{n \rightarrow \infty} \operatorname{Prob}(K(M)=0)=1-\frac{1}{e} \approx 63.2 \% .
$$

Proof. Let $R$ be a random binary variable such that $R=1$ represents the success to be a local optimum and $R=0$ the corresponding failure, with the success probability

$$
p=\operatorname{Prob}(R=1)=\frac{1}{2^{n}}=\frac{1}{M} .
$$

For Lemma 3.2 we have that if $M$ is large enough or, more precisely, when $K(M) \ll \# X=M=2^{n}$, the events $R=1$ are close to being independent. Also, as $M$ goes to infinity the probability $p$ goes accordingly to zero and the product $\lambda \equiv p M$ remains constant and equal to 1 . Hence by the law of small numbers (cf. Theorem 2 of Arratia et al., 1989; Falk et al., 2004) the distribution of $K(M)$ converges to Poisson distribution with mean $\lambda=1$ in (3), which is our thesis.

The result stated above generalizes the numerical results obtained in Amendola, Marengo, and Settepanella (2012).

Remark 3.4. By Theorem 2 of Arratia et al. (1989) it can be shown that

$$
\left|\operatorname{Prob}(K(M)=0)-\frac{1}{e}\right| \leq \frac{2 n}{2^{n}-1}
$$

for all $n \geq 1$. Hence for $n \geq 10$, a locally optimal outcome is actually reached in more than $60 \%$ of the cases.

Moreover, the above result does not only apply to local optima. In what follows we show that $\mathrm{u}$-local optima are asymptotically indistinguishable from local optima, in the sense that they have the same asymptotic distribution. 
Theorem 3.5. Let $\operatorname{Prob}_{n}\left(u_{z}\right)$ be the probability that a randomly chosen $z$ is a u-local optimum. Thus:

$$
\lim _{n \rightarrow \infty} \frac{\operatorname{Prob}_{n}\left(u_{z}\right)}{\operatorname{Prob}_{n}(z)}=1
$$

As a consequence Theorem 3.3 applies also for u-local optima.

Proof. By definition of u-local optimum, a free social outcome $z \in X$ is an u-local optimum if and only if for any social outcome $x \in X$ it exists a domination path starting from $x$ and ending up in $z$. The probability $\operatorname{Prob}_{n}\left(u_{z}\right)$ for $z$ to be a u-local optimum is thus given by:

$$
\operatorname{Prob}_{n}\left(u_{z}\right)=\operatorname{Prob}_{n}(z) \cdot \operatorname{Prob}_{n}\left(U_{z}\right) \text {, }
$$

where $\operatorname{Prob}_{n}\left(U_{z}\right)$ is the probability that a graph with $M$ nodes has a domination path starting from $x$ and ending up in $z$, for each $x \in X$.

As remarked in Section 1, a graph is irreducible if and only if each pair of nodes is contained within a cycle. It is an easy remark that if there is a cycle that involves both $x$ and $z$, then there is a domination path starting from $x$ and ending up in $z$. Hence the probability $\operatorname{Prob}_{n}\left(U_{z}\right)$ is greater than the probability $\operatorname{Prob}(M)$ that a tournament with M nodes is irreducible.

In Section 1 we have seen that Prob $(M)$ tends to 1 when $M$ goes to infinity. As a simple, but important, consequence, we have that when the number of social outcomes $M$ is big enough (and hence for large $n$ ) then a local optimum is a u-local optimum, which is our thesis.

As for local optima this result is in agreement with the numerical analysis presented in Amendola, Marengo, and Settepanella (2012).

A remarkable consequence of the results presented in this section is that the decision in a group is independent from the status quo. Indeed an agent who has the power to choose the object scheme has the power to achieve his or her preferred choice independently from the status quo, just changing the object scheme whenever a status quo is chosen.

\section{Conclusions}

In Amendola, Marengo, and Settepanella (2012) it is conjectured that the voting model introduced by MAST is highly decidable, i.e. a group of agents 
that agrees to use such voting process (linking each alternative to a yes/no preference) has a high probability to reach a final decision. In this paper we have proved that this conjecture is true and, further, that the final decision is independent from the initial condition, i.e. that the initial status quo does not affect the outcome of the social choice process.

The independence of local optima from the agenda (as stated in MAST) together with our results (high probability of having a local optimum and independence from the status quo), proves that the MAST model provides a useful analytical and numerical tool suitable for analyzing questions of social decisions, power, as well as consumer choice over complex bundles of product characteristics.

The computation of the probability of having an optimum for an a priori bundling of objects is the natural completion of the results presented here and is left for future research.

\section{References}

Amendola, G., L. Marengo, and S. Settepanella (2012). Decidability and manipulability in social choice. Working Paper LEM.

Amendola, G. and S. Settepanella (2012). Optimality in social choice. The Journal of Mathematical Sociology 36(1), 44-77.

Arratia, R., L. Goldstein, and L. Gordon (1989). Two moments suffice for Poisson approximations: the Chen-Stein method. Ann. Probab. 17(1), $9-25$.

Arrow, K. J. (1951). Social Choice and Individual Values. Yale University Press.

Chartrand, G. and L. Lesniak (2005). Graphs and Digraphs. Champan and Hall/CRC, Boca Raton, FL.

Denzau, A. T. and R. J. Mackay (1981). Structure-induced equilibria and perfect-foresight expectations. American Journal of Political Science 25, 762-779.

Elster, J. and A. Hylland (1986). Foundations of Social Choice Theory. Cambridge University Press. 
Enelow, J. M. and M. J. Hinich (1983). Voting one issue at a time: The question of voter forecasts. The American Political Science Review 77, 435-445.

Falk, M., J. Hü, and R. D. Reiss (2004). Laws of Small Numbers: Extremes and Rare Events. Birkhäuser.

Feldman, A. M. and R. Serrano (2006). Welfare Economics and Social Choice Theory, II Edition. Springer.

Gaertner, W. (2006). A Primer in Social Choice Theory. Oxford University Press.

Kramer, G. H. (1972). Sophisticated voting over multidimensional choice spaces. Journal of Mathematical Sociology 2, 165-180.

Marengo, L. and S. Settepanella (2010). Social choice among complex objects. Worging Paper LEM.

Moon, J. W. (1968). Topics on tournaments. Holt, Rinehart and Winston, New York-Montreal, Que.-London.

Shepsle, K. A. (1979). Institutional arrangements and equilibrium in multidimensional voting models. American Journal of Political Science 23, $27-59$.

Simon, H. A. (1982). The Sciences of the Artificial. MIT Press, Cambridge, MA, 2nd ed.

Taylor, A. D. (2005). Social Choice and the Mathematics of Manipulation. Cambridge University Press. 